\title{
Ausschreibung Preise der DRG
}

\section{Wilhelm-Conrad-Röntgen- Preis}

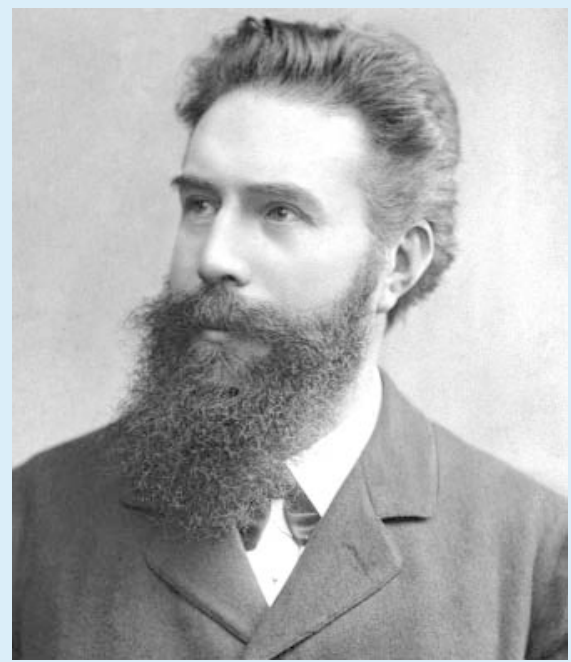

Prof. Dr. Dr. h. c. Wilhelm Conrad Röntgen (1845-1923). Entdeckung der nach ihm benannten Strahlen; Nobelpreisträger für Physik 1901; Ordinarius für Experimentalphysik in Gießen, Würzburg und München. Copyright: Archiv Deutsches Röntgenmuseum.

Zum Gedenken an ihren Namensgeber vergibt die Deutsche Röntgengesellschaft seit 1979 jährlich den Wilhelm-Conrad-Röntgen-Preis. Er ist mit 10.000 Euro dotiert, die Preisverleihung findet während des Deutschen Röntgenkongresses statt.

\section{Auswahlkriterien}

Der Preis wird verliehen für eine hervorragende wissenschaftliche Arbeit, die dem Fortschritt der Radiologie dient. Eingeschlossen sind die Arbeitsgebiete Radiologische Diagnostik, Strahlentherapie, Strahlenphysik, -biologie und -technik. Die Arbeit muss in deutscher oder englischer Sprache abgefasst sein und sie sollte noch nicht ausgezeichnet worden sein.

An der eingereichten Arbeit dürfen nicht mehr als drei Autoren beteiligt sein. Der oder die Autoren sollten zudem nicht älter als 40 Jahre alt sein, und sie müssen Mitglieder der Deutschen Röntgengesellschaft oder einer anderen der Europäischen Gesellschaft für Radiologie (ESR) angehörenden nationalen Gesellschaft für Radiologie sein.

\section{Bewerbungsunterlagen und Fristen}

Die Bewerbungsunterlagen inklusive der wissenschaftlichen Arbeit, einer ausführlichen Zusammenfassung der Arbeit, Lebenslauf mit beruflichem Werdegang, Lichtbild und Schriftenverzeichnis per E-Mail in elektronischer Form (PDF-Dokument) bis zum 19.11.2019 einzureichen an Frau Ermisch (DRG-Geschäftsstelle):

\section{E-Mail: ermisch@drg.de}

\section{Marie-Curie-Ring}

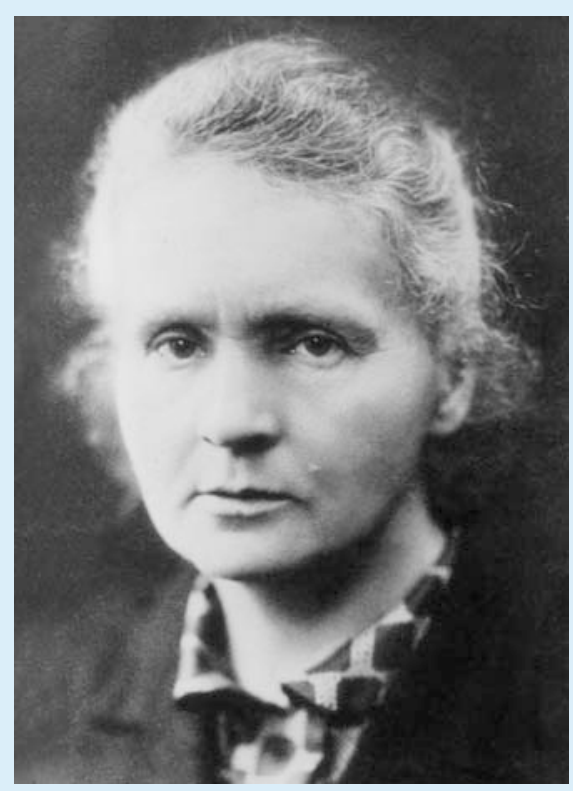

Marie Curie (1867-1934). Entdeckung der chemischen Elemente Radium und Polonium, Prägung des Begriffs Radioaktivität; Nobelpreisträgerin für Physik (1903) und Chemie (1911); Lehrstuhl Institut für Allgemeine Physik an der Sorbonne, Paris. Copyright: Archiv Deutsches Röntgenmuseum.

Der zur 50-Jahrfeier der Deutschen Röntgengesellschaft gestiftete Hermann-Holthusen-Ring, der von 2010 bis 2014 Wilhelm-Conrad-Röntgen-Ring hieß, wurde 2015 nach der bedeutenden Atomphysikerin und Radiologin Marie Curie umbenannt. Die in Polen geborene und in Frankreich berühmt gewordene Nobelpreisträgerin (1867-1934) hat die Physik, Chemie und Radiologie gleichermaßen immens beeinflusst, insbesondere durch die von ihr ent- deckten Elemente Radium und Polonium, ihre Studien zur Radioaktivität und ihr Wirken während des 1 . Weltkriegs, als sie mit einem mobilen Röntgenwagen verletzte Soldaten direkt an der Front untersuchte.

Der Ring zeichnet einen herausragenden jungen Wissenschaftler der Radiologie aus, wobei die Kandidaten/innen hierfür vorgeschlagen werden müssen. Grundlage der Begutachtung sind die gesamten bisherigen Leistungen, die im besonderen Maße befähigen sollen, eine herausragende wissenschaftliche Laufbahn zu vollenden.

Mit der Umbenennung in Marie-Curie-Ring wird diese Perspektive beispielhaft hervorgehoben. Die Preisverleihung findet jährlich auf dem Deutschen Röntgenkongress statt. Eine Jury entscheidet über die Preisvergabe.

Das wichtigste Kriterium für die Auszeichnung sind die wissenschaftliche Reputation und Kompetenzen, die sich die Kandidaten/ innen im Rahmen von Veröffentlichungen und Vorträgen im nationalen und internationalen Rahmen erworben haben. Die Preisträger/innen sollten in der Regel nicht älter als 40 Jahre sein (bei Kandidatinnen verlängert sich diese Frist um jeweils zwei Jahre pro Kind) und werden vorgeschlagen.

\section{Bewerbungsunterlagen und Fristen}

- ausführliche Zusammenfassung der wissenschaftlichen Schwerpunkte und Entwicklungen auf 5 Seiten

- Lebenslauf mit beruflichem Werdegang

- Lichtbild

- Schriftenverzeichnis, gegliedert nach Originalarbeiten als Erst- und Letztautor, Koautor, Übersichtsarbeiten und Kasuistiken, Impact Faktor des Erscheinungsjahres bitte angeben

- Patente

- Drittmitteleinwerbungen

Bitte senden Sie Ihren Vorschlag mit den erbetenen Unterlagen in elektronischer Form per E-Mail an Frau Ermisch (DRG-Geschäftsstelle):

E-Mail: ermisch@drg.de 
Einreichungsfrist für den Marie-Curie-Ring 2020 ist der 31.01.2020.

Verleihung auf dem Deutschen Röntgenkongress.

\section{Walter-Friedrich-Preis}

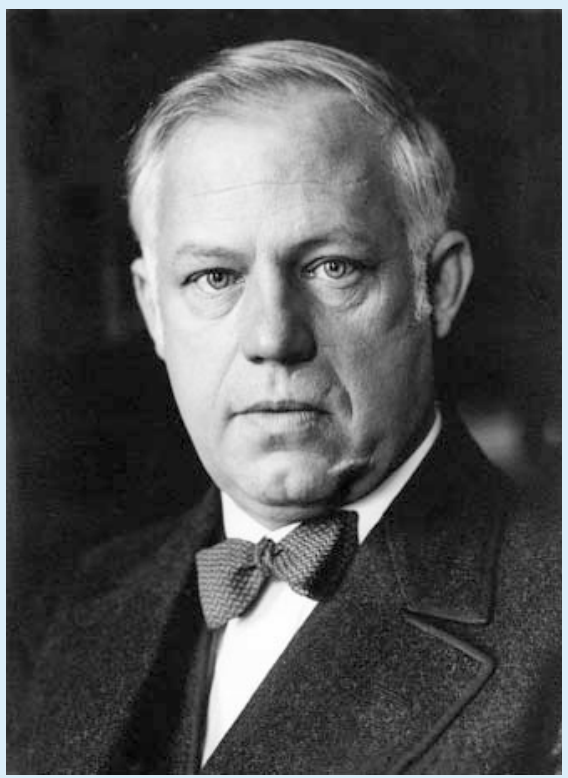

Prof. Dr. Walter Friedrich(1883-1969). Pionier der Strahlenphysik; Ordinarius für Medizinische Physik in Berlin 1922. Copyright: DRG.

Der Preis würdigt die wissenschaftlichen Leistungen und das prägende Wirken von Prof. Walter Friedrich für die medizinische Radiologie und Strahlentherapie. Das Stiftungsvermögen des Preises entstammt der Gesellschaft für Medizinische Radiologie der ehemaligen DDR und wurde 1992 der DRG übertragen, die das Vermögen 2003 durch eine Zustiftung erhöhte. Der Preis ist mit 2.500 Euro dotiert und wird jährlich während des Deutschen Röntgenkongresses verliehen.

\section{Auswahlkriterien}

Der Walter-Friedrich-Preis berücksichtigt insbesondere die Gemeinsamkeit von Physik, Technik, Biologie und Medizinischer Radiologie. Er wird verliehen für herausragende wissenschaftliche Arbeiten (incl. Habilitationsarbeiten) auf dem Gesamtgebiet der Radiologie, für beispielgebende Publikationen und Initiativen zur Verbesserung der Lehre auf allen (Teil-)Gebieten der Medizini- schen Radiologie und für vorbildliche praktische Ergebnisse in der Medizinischen Radiologie inkl. technischer Entwicklungen.

Bewerben können sich Einzelpersonen oder Arbeitsgruppen. Der/Die Bewerber sollte/n Mitglied/er der Deutschen Röntgengesellschaft oder einer anderen der ESR angehörenden nationalen Gesellschaft für Radiologie sein und bei Einreichung der Preisarbeiten das 40. Lebensjahr nicht vollendet haben.

\section{Bewerbungsunterlagen und Fristen}

Die Bewerbungsunterlagen sind per E-Mail in elektronischer Form (PDF-Dokument) bis zum 19.11.2019 einzureichen an Frau Ermisch (DRG-Geschäftsstelle):

E-Mail: ermisch@drg.de

\section{Alfred-Breit-Preis}

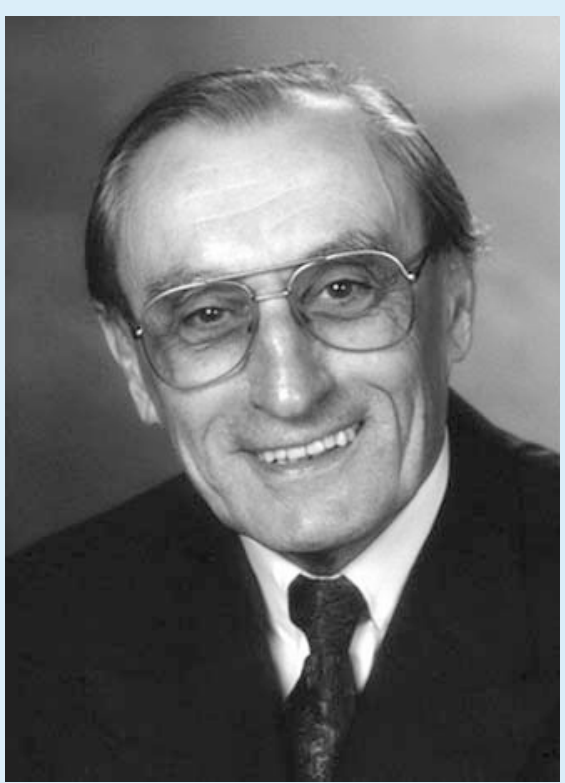

Prof. Dr. med. Alfred Breit (1922-2011).

Copyright: DRG

Der Preis erinnert an Prof. Alfred Breit, einen der Wegbereiter der Strahlentherapie. Der Preis wird seit 2012 jährlich vergeben und ist mit 20.000 Euro dotiert. Die Verleihung findet während des Deutschen Röntgenkongresses statt.

\section{Auswahlkriterien}

Die Kriterien für den Alfred-Breit-Preis sind testamentarisch festgelegt. Der Preis wird für Arbeiten und Entwicklungen vergeben, die aus dem Bereich der radiologischen Forschung kommen und erkennbar zu Fortschritten in der Krebstherapie beitragen können. Als Grundlage zur Entscheidung für die Preisvergabe können Publikationen oder Patente dienen, aber auch Verfahren, die sich in der Klinik etabliert haben, ohne dass sie publiziert worden sind.

Der Preis kann an einzelne Wissenschaftler, aber auch an Arbeitsgruppen oder Institutionen vergeben werden. Kandidaten können auch von Dritten vorgeschlagen werden; Voraussetzung hierfür ist ein abgeschlossenes wissenschaftliches Studium bzw. eine entsprechende abgeschlossene Ausbildung der/des Kandidatin/Kandidaten.

\section{Bewerbungsunterlagen und Fristen}

- Eine kurze Zusammenfassung der Arbeit/des Patents/des klinischen Verfahrens (max. 2 DIN A4-Seiten)

- Eine Kopie der Publikation(en)/des Patents bzw. eine ausführliche Beschreibung des für den Preis vorgeschlagenen Verfahrens

- einen Lebenslauf des Erstautors/der Erstautorin bzw. des Leiters/der Leiterin der Arbeitsgruppe bzw. des Patentinhaber/der Patentinhaberin

- ein Publikationsverzeichnis derselben Person

- bei Bewerbungen von Einzelpersonen: ein Lichtbild

Die Bewerbungsunterlagen sind per E-Mail in elektronischer Form (PDF-Dokument) bis zum 31.01.2020 an Frau Ermisch (DRGGeschäftsstelle) einzureichen:

E-Mail: ermisch@drg.de

\section{Promotionspreis der}

\section{Deutschen}

\section{Röntgengesellschaft}

Der Promotionspreis der Deutschen Röntgengesellschaft wurde erstmals zum Jahr 2012 ausgeschrieben, um wissenschaftlich engagierte Studenten und Mediziner zu motivieren und zu fördern. Pro Jahr werden bis zu drei Promotionsarbeiten ausgezeichnet. Im Fall einer Auszeichnung der eingereichten Arbeit wird diese als Originalarbeit in der Fachzeitschrift RöFo veröffentlicht. 
Der Preis ist mit 1.000 Euro und der kostenIosen Teilnahme am kommenden Deutschen Röntgenkongress dotiert.

\section{Auswahlkriterien}

Teilnehmen können Bewerber, die im vorhergehenden Jahr auf dem Gebiet der Radiologie und Medizinphysik promoviert haben. Die eingereichte Arbeit resultiert aus der Promotion (bzw. aus Teilen der Promotion) und ist in Form einer wissenschaftlichen Originalarbeit nach den Richtlinien für Autoren der RöFo abgefasst. Von einer Fachzeitschrift bereits veröffentlichte oder zur Publikation angenommene Arbeiten, die aus der Promotion des Bewerbers resultieren, können ebenfalls für den Preis eingereicht werden. Sie werden, falls sie für den Preis ausgewählt werden, nach ihrer Veröffentlichung in der RöFo referiert.

\section{Bewerbungsunterlagen und Fristen}

Die Bewerbungsunterlagen enthalten die wissenschaftliche Arbeit, einen kurzen Lebenslauf und ein Lichtbild. Sie sind per E-Mail in elektronischer Form (PDF-Format) bis zum 19.11.2019 an Frau Ermisch (DRG-Geschäftsstelle) einzusenden:

E-Mail: ermisch@drg.de

\section{Young Investigator Award}

Auf Initiative der AG Methodik und Forschung werden seit dem Jahr 2009 alljährlich anlässlich des Deutschen Röntgenkongresses mit dem Young Investigator Award herausragende Arbeiten und Kongressbeiträge junger Wissenschaftler geehrt. Mit dem YIA sollen die Leistungen des wissenschaftlichen Nachwuchses in der Bildgebung herausgehoben und gefördert werden. Die Tatsache, dass von den bisherigen Preisträgern mehr als die Hälfte Naturwissenschaftler waren, mag als Zeichen dafür gesehen werden, dass der Award vor allem sehr innovative Forscher anlockt. Der Preis ist mit 500 Euro dotiert.

\section{Auswahlkriterien}

Für den YIA können sich Wissenschaftler unter 35 Jahren mit ihren Arbeiten im Rahmen der regulären Abstracteinreichung bewerben. Die für den YIA eingereichten Abstracts sollen in Form und Struktur den Kriterien für reguläre Beiträge zum Deutschen Röntgenkongress folgen. Alle für den YIA eingereichten Abstracts durchlaufen zunächst das reguläre Reviewverfahren der wissenschaftlichen Kongressbeiträge. Anschließend erhält die YIAJury jeweils die besten zwei für den YIA eingereichten Abstracts aus allen Themengebieten und wählt hieraus insgesamt sechs Beiträge für die YIA-Session aus. Diese sechs Beiträge werden im Rahmen der YIA Session beim Deutschen Röntgenkongress vorgestellt. In den dedizierten YIA Sessions stellen die jungen Wissenschaftler ihre Arbeit einer Jury renommierter deutscher Wissenschaftler vor, nach deren Votum der Preis verliehen wird.

\section{Bewerbung}

Die Bewerbung erfolgt im Rahmen der regulären Abstracteinreichung für den Deutschen Röntgenkongress. 